\title{
Micromechanical models for stiffness prediction of alumina trihydrate (ATH) reinforced poly (methyl methacrylate) (PMMA): effect of filler volume fraction and temperature.
}

O. A. Stapountzi, M. N. Charalambides, J. G. Williams

Mechanical Engineering Department, Imperial College London SW7 2AZ, UK

The elastic modulus of alumina trihydrate (ATH) reinforced poly (methyl methacrylate) was measured at different filler volume fractions and temperatures. The experimental values showed a $35 \%$ increase as the filler contents increased from $33 \%$ vol. to $49 \%$ vol., and a $50 \%$ decrease with rising temperature from 0 to $90{ }^{\circ} \mathrm{C}$. The findings were confirmed by analytical modelling predictions from the literature. Four analytical models were in very good agreement with the experimental data, including the Halpin-Tsai, the Generalised Self Consistent, the Lielens and Nielsen models. Amongst them the best model for the volume fractions used here was that of Lielens.

Keywords: A. Polymer matrix composites, A. Particle reinforced composites, B. Mechanical properties, C. Modelling.

\section{Introduction}

Composites made from alumina trihydrate (ATH) filled poly (methyl methacrylate) (PMMA) are being used as engineering materials in structural applications because of their enhanced properties and low production costs [1]. They are usually made with high volume fractions of filler to give a large increase in the elastic modulus which is important in several applications. It is useful to know if these increases can be predicted from the large number of theories available [2-4]. Some of the theories are limited to low volume fraction composites.

There is some interest in predicting the effects of temperature on modulus for design purposes. However there is also an incentive to measure the properties over a range of 
temperatures because the matrix values change and can be measured separately. The properties of the filler do not change. Thus when materials with varying volume fractions of filler are tested over a range of temperatures, they provide data over a very wide range of matrix property values which can be compared to predictions from the various theories.

\section{Analytical models}

The theories dealing with the prediction of the elastic performance of two-phase composites can be classified into two main groups: constitutive relationships and semiempirical equations. They all consist of analytical expressions that relate the effective moduli of composite materials to the volume fractions and the elastic moduli of their constituents, i.e., the matrix and particle inclusions. The matrix and inclusions are assumed to be linearly elastic and homogeneous, the matrix is isotropic, the particles isotropic or transversely isotropic and identical in shape and size. The subscripts, $m$ and $f$, are used to denote the matrix and filler particles (inclusions). For each model discussed below, only the relevant equations will be outlined with no details on their derivation for brevity purposes. For more details on the specific models, the reader is directed to the original publications or [4].

\subsection{Constitutive relationships}

The basis of almost all existing constitutive models for elastic modulus prediction of particle filled composites lies in Hill's theory [5] for average stress and strain in a representative volume $V_{f}$ of the two-phase composite material. In the representative volume concept, $V_{f}$ represents the volume of the inclusions and $V_{m}$ the volume of the matrix, such that $V=V_{f}+V_{m}$. The constituent volume fractions $f_{f}$ and $f_{m}$ can be expressed by Eqn. 1:

$f_{f}=V_{f} / V, \quad f_{m}=V_{m} / V \quad$ and $\quad f_{f}+f_{m}=1$ 
The fundamental expression for the effective elastic stiffness of composite materials is [5]:

$$
\boldsymbol{C}=\boldsymbol{C}_{m}+f_{f}\left(\boldsymbol{C}_{f}-\boldsymbol{C}_{m}\right) \boldsymbol{A}_{f}
$$

where $\boldsymbol{C}_{m}$ and $\boldsymbol{C}_{f}$ are the elastic stiffness tensors for the matrix and inclusions respectively and $\boldsymbol{A}_{f}$ is the strain-concentration tensor which relates the average strain in the inclusions, $\bar{\varepsilon}_{f}$, with the average strain in the representative volume, $\bar{\varepsilon}$, through:

$e_{f}^{-}=A_{f} \bar{e}$

The theoretical prediction of the elastic components is dependent upon the determination of the strain concentration tensor of the inclusions, $\boldsymbol{A}_{f}$. The first attempt to characterise the complicated strain fields occurring in and around an inclusion contained in an infinite matrix was provided by Eshelby [6]. Eshelby considered the case of a single inclusion embedded in an infinite medium which is subjected to conditions of uniform deformation at large distances from the inclusion. Hill earlier showed that the average strain within the representative volume is identical to the uniform strain applied on the surface of the volume, $\varepsilon_{o}$, i.e., $\bar{\varepsilon}=\varepsilon_{o}$ (average strain theorem). Eshelby used this theorem to show that the strain concentration factor, $\boldsymbol{A}_{f}=\overline{\varepsilon_{f}} / \boldsymbol{\varepsilon}_{\boldsymbol{o}}=\boldsymbol{A}_{S}$, of an ellipsoidal inclusion surrounded by an infinite matrix subjected to uniform deformation, i.e., $\boldsymbol{u}(\boldsymbol{S})=\boldsymbol{\varepsilon}_{\boldsymbol{o}} \boldsymbol{x}$, at long distances from the inclusion, is expressed by:



where $\boldsymbol{S}$ stands for the Eshelby's tensor. Expressions for the components of the Eshelby's tensor, $\boldsymbol{S}$, in terms of the matrix Poisson's ratio, $n_{m}$, and the aspect ratio, $a$, of spheroidal inclusions shown in Fig 1, can be found in [7]. 
Eqn. 4 can be combined with Eqn. 2, to predict the components of the elastic stiffness tensor for composites filled with spherical or spheroidal inclusions at dilute concentrations.

The Mori-Tanaka (M-T) theory [9] is based on the combination of the average uniform strain inside an ellipsoidal inclusion due to Eshelby, and the theory of the average stress in the matrix [9]. The M-T strain concentration tensor is obtained by:

$$
\left.\boldsymbol{A}_{M-T}=\boldsymbol{A}_{S} \dot{g}^{\left(1-f_{f}\right.}\right) \boldsymbol{I}+f_{f} \boldsymbol{A}_{S} \stackrel{\mathrm{u}}{\mathrm{u}}^{1}
$$

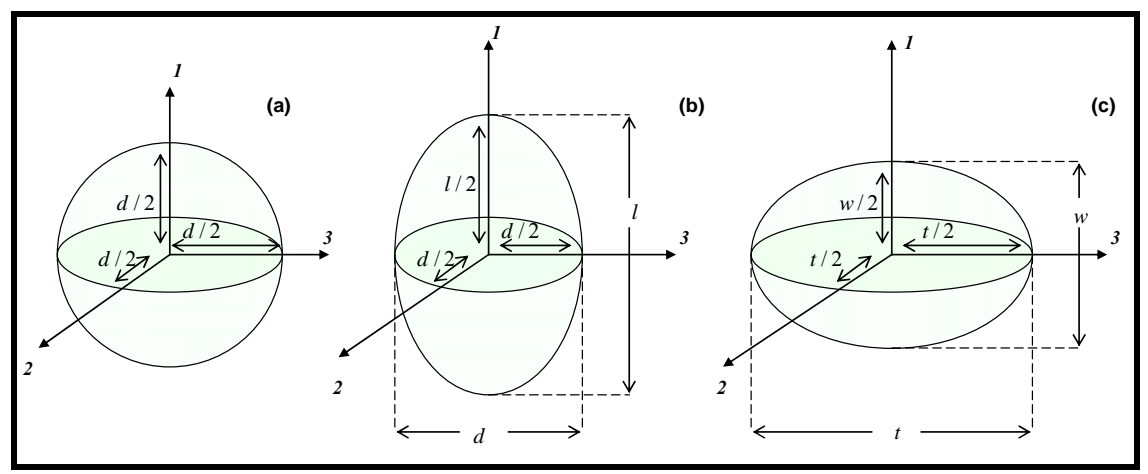

Fig. 1 (a) Spherical inclusions (isotropic) $a=1$, (b) fibre-like (prolate spheroid) inclusions, $a=l / d$, and (c) disk-like (oblate spheroid) inclusions, $a=t / w ;(\mathrm{b})$ and (c) are transversely isotropic in the 2-3 plane.

The M-T mean-field theory has been effectively applied to study the elastic behaviour of fibre-reinforced composites at relatively low volume ftractions.

To overcome the complications associated with the description of the internal microstructure of composite materials, many authors have concentrated on finding the upper and lower bounds of the elastic moduli. The main feature of the bounding techniques is the evaluation of the energy content of the materials undergoing deformation, which is in turn associated with an assumed stress/strain field in the composite systems [10]. Variational principles of the elasticity theory, e.g., minimum or maximum stress/strain conditions and minimum energy assumptions are employed in such models. The simplest 'bounding' models 
are the Voigt and Reuss [11] approximations, known as the 'rule of mixtures' and the 'inverse rule of mixtures', respectively.

Closer bounds for the elastic moduli have been developed for continuous fibre composites by Hill $[5,12]$ and Hashin and Shtrikman [8]. The Hashin-Shtrikman approach is based on the introduction of a homogeneous and isotropic reference material. The method is based on evaluating the differences between the 'comparison' material and the actual composite. In order to calculate the upper bounds, the 'comparison' material is chosen to have stiffness values higher than or equal to the rigidity of the inclusions (hard phase), and for the lower bounds the reference material has stiffness smaller than or equal to the stiffness of the matrix (softer phase).

Weng [13] extended the Hashin and Shtrikman method and obtained detailed expressions for the elastic stiffness tensor of aligned ellipsoidal inclusions. By choosing the matrix or filler as reference material, and with the use of the 'convenient' strain concentration factor $\boldsymbol{M}$, ie:

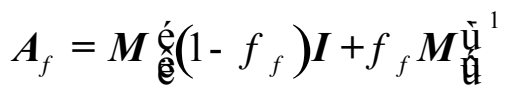

the lower bound of the 'convenient' strain concentration factor, $\boldsymbol{M}_{\text {lower }}$, is:

$$
\boldsymbol{M}_{\text {lower }}=\frac{e}{\mathrm{e}}+\boldsymbol{S}_{m} \boldsymbol{C}_{m}^{-1}\left(\boldsymbol{C}_{f}-\boldsymbol{C}_{m}\right)_{\mathrm{e}}^{\mathrm{u}^{1}}
$$

and the upper bound of the 'convenient' strain concentration tensor $\boldsymbol{M}_{\text {upper }}$, is:

$$
\boldsymbol{M}_{\text {upper }}=\mathrm{e}_{\mathrm{e}}^{\mathrm{I}}+\boldsymbol{S}_{f} \boldsymbol{C}_{f}^{-1}\left(\boldsymbol{C}_{m}-\boldsymbol{C}_{f}\right)_{\mathrm{i}}^{\mathrm{u}^{1}}
$$

The 'convenient' strain concentration tensors, $\boldsymbol{M}_{\text {lower }}$ and $\boldsymbol{M}_{\text {upper }}$ are substituted into Eqn 6 to derive the lower and upper bound strain concentration tensors $\boldsymbol{A}_{\text {lower }}$ and $\boldsymbol{A}_{\text {upper }}$. The latter can then be substituted in Eqn 2 to derive lower and upper bound values for the stiffness tensors of the composite. The lower bounds obtained using the above considerations coincide 
with the predictions of the M-T theory. The upper bounds predicted by Eqns 6, 2 and 8 represent precise estimates of the composite moduli for very high particle concentrations, i.e., at maximum particle packing conditions. This observation, inspired Lielens et al. [14], to use a "mixture rule between the upper and lower bound" to provide predictions of the elastic moduli of composites with intermediate filler volume fractions. The Lielens 'convenient' strain concentration factor $\boldsymbol{M}_{L}$ is given by

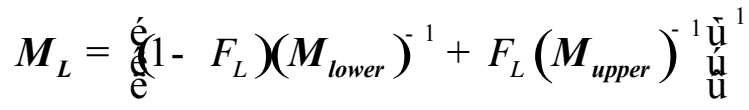

where the mixture function, $F_{L}$, must increase monotonously with the volume fraction of the inclusions, therefore for $f_{f}=0, F_{L}=0$ and for $f_{f}=1, F_{L}=1$. Lielens et al., suggested the following for the mixture function:

$$
F_{L}=\frac{f_{f}+f_{f}^{2}}{2}
$$

Eqns 9 and 10 give improved results at reinforcement volume fractions in the $40-60$ $\%$ vol. range.

The last class of constitutive models that are considered in this work are the ones based on the self-consistent method. In the self-consistent method, proposed by Hill [15], a single inclusion of ellipsoidal geometry is supposed to be embedded in an infinite homogeneous medium, the latter possessing the effective properties of the composite. Therefore self-consistent scheme assumes that the inclusion is in fact embedded in a composite material with unknown properties, rather than the matrix. From Eqn. 4, the selfconsistent concentration tensor is given by substituting the matrix tensor, $\boldsymbol{C}_{m}$, with the corresponding elastic tensor of the composite material, $\boldsymbol{C}$, yielding:

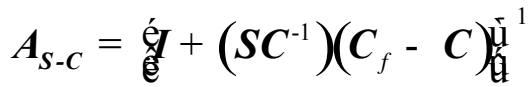


The combination of Eqns 11 and 2 provides the basic self-consistent formula for the overall stiffness. The terms $\boldsymbol{C}$ and $\left(\boldsymbol{C}^{-1}\right)$ in Eqn. 11, represent properties of the infinite medium and they are initially unknown $[2,15]$. The self-consistent models have often been found to over-predict the elastic moduli of particle filled composites, especially at higher volume fractions [2]. Therefore, another approach has been developed, called the generalized self-consistent (GSC) theory. It was proposed by Kerner [16] for spherical particles and by Hermans [17] for cylindrical fibre composites. The GSC scheme also uses an embedding method, except that in this case the inclusion is embedded in the matrix material; these concentric volumes of inclusion and matrix are themselves embedded in the composite medium [4]. For fillers that are much more rigid than the polymer matrix ( $G_{f} \square G_{m}$, where $G$ is shear modulus), Kerner's equation is reduced to:

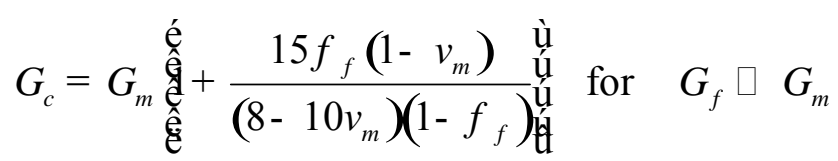

for up to intermediate filler volume fractions [1]. Eqn. 12 has been used in various studies to predict the elastic modulus of particle filled polymers directly by substitution of the term $G_{c}$ with $E_{c}$.

\subsection{Semi-empirical equations}

The results offered by Kerner and Hermans have been the foundation of the HalpinTsai (H-T) semi-empirical equations [18]. Halpin and Tsai discovered that Herman's and Kerner's equations could be expressed in a compact form as:

$$
\frac{P_{c}}{P_{m}}=\frac{1+s h f_{f}}{1-h f_{f}} \quad h=\frac{P_{f} / P_{m}-1}{P_{f} / P_{m}+s}
$$

where $f_{f}$, is the filler volume fraction, and $P$ represents the bulk and shear moduli while $s$, is a measure of the reinforcement packing geometry and loading conditions. For $s \quad{ }^{\circledR} 0$, the 
$\mathrm{H}-\mathrm{T}$ equations reduce to the 'inverse rule of mixtures', while for $s{ }^{\circledR} ¥$, the $\mathrm{H}-\mathrm{T}$ theory gives the Voigt upper bound. Assuming that the particles are aligned with the loading direction, Halpin and Kardos [19] proposed $s=2 a$ in order to compute the longitudinal modulus of short-fibre systems. The modulus in the transverse direction was found not to be sensitive to the aspect ratio with $s=2$.

The H-T equations have been found to under-predict the stiffness values of composites with relatively high filler loadings $[2,20]$. This has been credited to the fact that Herman's solution for continuous fibres does not consider any limit in maximum packing fraction in an actual composite system. To account for this maximum, Lewis \& Nielsen [20] proposed a modification to the original form of the $\mathrm{H}-\mathrm{T}$ equations, by introducing a new parameter $y\left(f_{f}\right)$ in Eqn 13:

$\frac{P_{c}}{P_{m}}=\frac{1+s_{N} h f_{f}}{1-h y\left(f_{f}\right) f_{f}} \quad h=\frac{P_{f} / P_{m}-1}{P_{f} / P_{m}+s_{N}}$

where $y\left(f_{f}\right)$ is a function of the volume fraction of the inclusions and the maximum packing of the inclusions ; $s_{N}=k_{E}-1$, with $k_{E}$ being the Einstein's coefficient. The values of $k_{E}$ and consequently $s_{N}$, are related to the bonding between filler and matrix [21]. For a matrix with $v_{m}=0.5$, filled with dispersed spheres, and if there is no slippage in the interface, the coefficient is $k_{E}=2.5,\left(k_{E}=1\right.$, if there is slippage). For $v_{m} £ 0.5$, the $k_{E}$ values are reduced and the shape factor $s_{N}$ is calculated directly by:

$s_{N}=\frac{7-5 v_{m}}{8-10 v_{m}}$

An empirical formula is proposed by Lewis and Nielsen [20] for the factor $y\left(f_{f}\right)$ as:

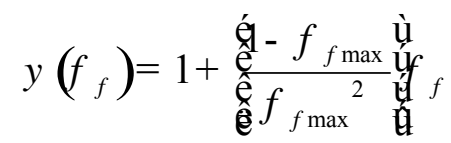


Nielsen and Landel [21] provide tabulated values for a range of particle shapes and packing fractions, e.g. $f_{f \max }=0.74$ for dispersed spheres with hexagonal close packing.

\subsection{Orientation of non-spherical inclusions}

In all of the above theories it is assumed that the inclusions are aligned. However, realistically all composites contain some level of filler disorientation. Composites with a random orientation of particles would be expected to give lower modulus values. Van Es et al. [22] used the laminate theory to provide simple approximations for the stiffness of composites with randomly oriented fibre-like or disk-like inclusions. The modulus of a short fibre reinforced composite with 3D randomly oriented fibres was generated as:

$$
E_{3 D \text { fibre }} @ 0.184 E_{1}+0.816 E_{2}
$$

where $E_{1}$ and $E_{2}$ are the longitudinal and transverse moduli respectively. A

unidirectional plate reinforced with disk-like particles, is already 2D randomly orientated and in this case the modulus of the composites is derived as:

$$
E_{3 D \text { disk }} @ 0.49 E_{1}+0.51 E_{2}
$$

\section{Experimental}

The composite materials used in the experiments were supplied by E.I. DuPont Nemours \& Co. (Inc.) in the form of sheets. The matrix constituent was slightly cross-linked PMMA. The plaques were all made with the same ATH filler with volume fractions ranging from $33 \%$ vol. to $49 \%$ vol. The degree of functionality and degree of cross-linking of the polymeric matrix formed during the casting process at each filler level was kept constant. To improve the interface between filler and matrix, the manufacturer used an adhesion promoting additive. Unfilled PMMA sheets were tested to provide the elastic properties of the matrix. The ATH particles were studied both as powder and as contained in the composites. Gibbsite 
was supplied by Alcan Chemicals USA, Division of Alcan Aluminium Corporation. The mechanical tests were performed for the nominal temperature range of $0{ }^{\circ} \mathrm{C}-90^{\circ} \mathrm{C}$.

Three-point bend tests were carried out for the composite materials and for unfilled PMMA. The tests were performed according to Method I - Procedure A of the ASTM D790M-93 standards for plastics [23], utilising simple beam specimens with rectangular cross sections. The thickness of the specimens was taken equal to the thickness of the material sheets, ie $12 \mathrm{~mm}$, and the width and length were $22.5 \mathrm{~mm}$ and $200 \mathrm{~mm}$ respectively. The rate of crosshead motion for each group of materials was set at $5.6 \mathrm{~mm} / \mathrm{min}$ computed according to the standards, where the strain rate is $0.01 \mathrm{~min}^{-1}$. A minimum of five replicates were used for each test.

Tensile tests were performed for the estimation of the elastic modulus and Poisson's ratio of unfilled PMMA. Tensile tests were also conducted on composites along perpendicular directions of the material sheets to exclude potential directional dependence of their elastic modulus. The tests were performed according to the ASTM D638M-84 standard [24] for plastics. For PMMA, the tests were carried out using Type M-I tensile 'bone-shaped' specimens with a thickness of $7.5 \mathrm{~mm}$ and gauge length and width equal to $50 \mathrm{~mm}$ and 10 mm respectively. For the composites, Type M_II tensile specimens were used with a thickness of $4 \mathrm{~mm}$ and gauge length and width equal to $25 \mathrm{~mm}$ and $6 \mathrm{~mm}$ respectively. The elastic modulus and Poisson's ratio of PMMA were measured in tension using metal-foil strain gauges. The test speed was set at $1 \mathrm{~mm} / \mathrm{min}$, resulting in strain rates of $0.02 \mathrm{~min}^{-1}$. For the composites tests, an extensometer was attached onto the surface of the samples to record the strain within the gauge length. The test rate was set at $1 \mathrm{~mm} / \mathrm{min}$, giving strain rates of $0.04 \mathrm{~min}^{-1}$.

Optical microscopy was used to provide an indication of the dispersion of particles into the polymer matrix and to characterise their shape, as they are contained in the 
composites. Samples with cross-sectional areas of $12 \mathrm{~mm}$ x $20 \mathrm{~mm}$ were cut from both the surfaces and through the thickness planes. A succession of polishing steps was followed which aimed to remove any damage produced by sectioning. A minimum number of ten images at random locations of each of the samples was acquired, and quantitative statistical measurements were performed using the image processing software ImageJ [25].

Scanning electron microscopy (SEM) was used to observe the morphology and shape characteristics of the ATH powder. To minimise charging of the samples, a thin layer of gold was deposited on the surfaces prior to observation.

Laser light scattering (LLS) was used to assess the aspect ratio of the particles and confirm the findings of the optical microscopy. A particle analyser, Malvern Mastersizer 2000 $(0.02 \mu \mathrm{m}$ to $2 \mathrm{~mm})$ was utilised. The particles were dispersed in distilled water, and the amount of water $(100 \mathrm{ml})$ and the quantity of powder $(159.5 \mathrm{mg})$ used were measured before and after the experiment. The ratio between the actual volume concentration presented to the measuring device and the volume concentration calculated by the Malvern Mastersizer was used to provide an estimate of the aspect ratio of the particles [26].

\section{Experimental results}

Fig. 2 shows the results of the flexural modulus of the composites plotted against filler volume fraction and temperature from 0 to $94{ }^{\circ} \mathrm{C}$. The standard deviation of the modulus values presented in the graphs is less than $2 \%$ of the mean values (five replicates, tested four times each). As they were so small compared to the symbol size, they are not shown.

The modulus for the composites shows a $35 \%$ increase as the filler content increases from $33 \%$ to $49 \%$ vol. Parallel trends of dependence of the elastic modulus on the amount of the filler are seen at all temperatures. The modulus of the composites decreases by $50 \%$ with rising temperature from 0 to $90{ }^{\circ} \mathrm{C}$.

The difference in the modulus values obtained from the tensile tests along 
perpendicular directions of the composite sheets was less than $5 \%$, indicating random orientation of the filler particles in the composites.

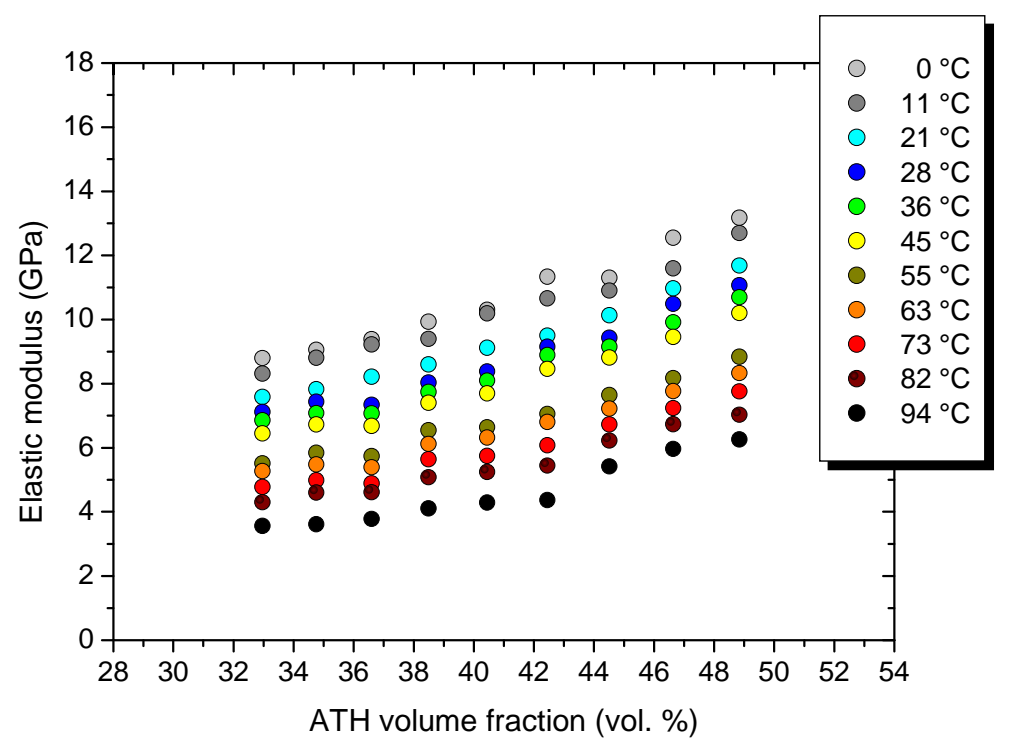

Fig. 2 Flexural modulus (GPa) vs. filler volume fraction for ATH-PMMA composites.

The results for the tensile modulus of PMMA are shown in Fig. 3, along with the values from the flexural tests. The tensile data presented by Ferreira et al. [27] for PMMA ( strain rate $=0.04 \mathrm{~min}^{-1}$ ) are given for comparison and verification of the results. Very good agreement is observed within the three sets of data.

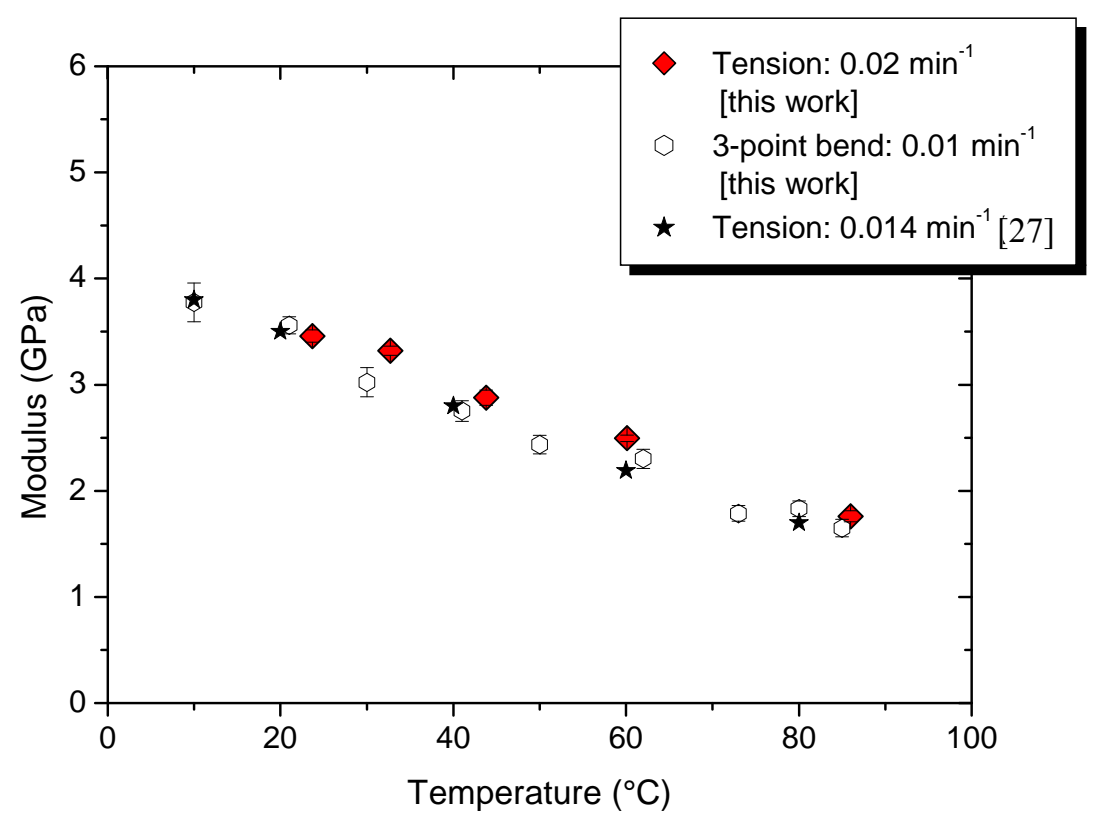

Fig. 3 Modulus of unfilled PMMA as a function of temperature. 
The Poisson's ratio data computed from the transverse vs. axial strain plots are presented in Fig. 4. The results are compared to the values calculated [4] from pressurevolume-temperature (PVT) data presented by Zoller and Walsh [28] on PMMA. Good agreement is observed between the results, with the Poisson's ratio of increasing gradually with temperature.

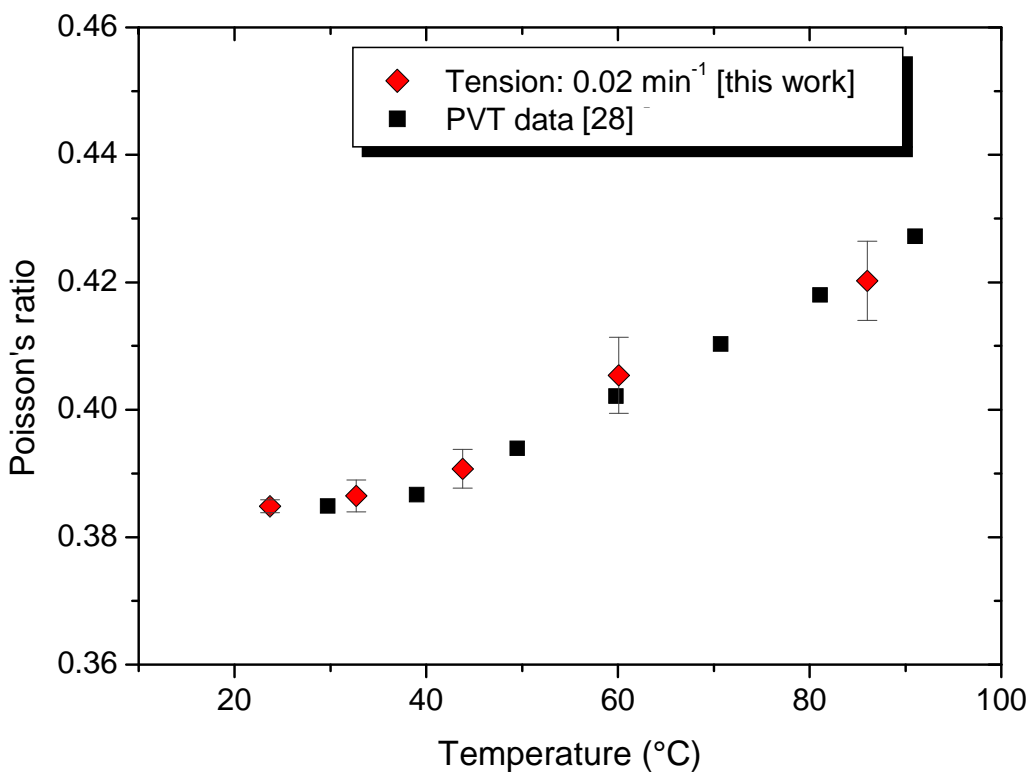

Fig. 4 Poisson's ratio of unfilled PMMA as a function of temperature

Typical optical micrographs of the ATH-PMMA surfaces containing filler loadings of $29 \%$ and $49 \%$ vol. are presented in Fig. 5. The lighter phase represents the ATH particles while the grey regions correspond to the PMMA matrix.

The digital photographs were converted into 8-bit greyscale images and transformed into binary images (see Fig 6a). The ImageJ facility 'shape descriptors' [25], was then utilised to obtain the aspect ratio of the particles. This is determined by fitting an ellipse to each particle and the aspect ratio is obtained from the ratio of the major to the minor axis (see Fig. $6 b)$. The calculations were based on the constituent areal fractions assuming that it was well represented by the regions shown in the optical pictures. 


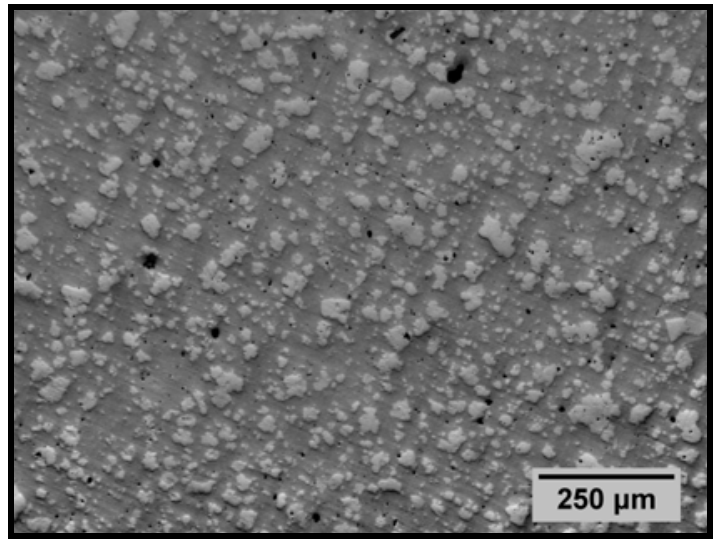

a.

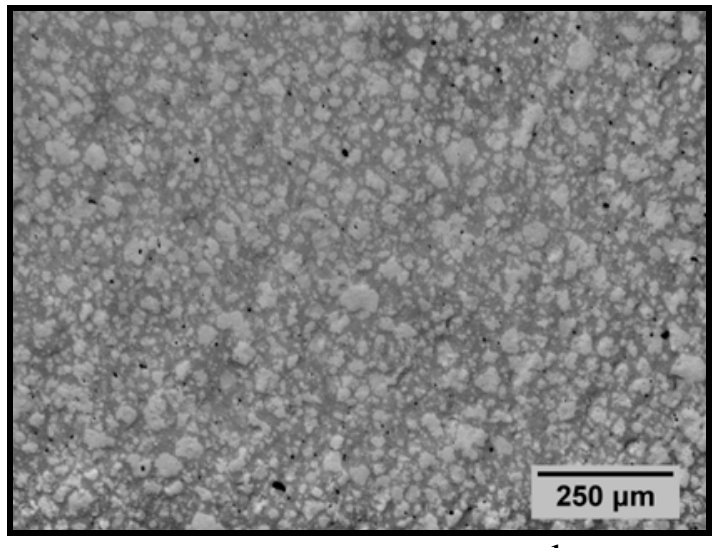

b.

Fig. 5 Typical optical micrographs of the ATH-PMMA a. $29 \%$ vol., b. $49 \%$ vol.

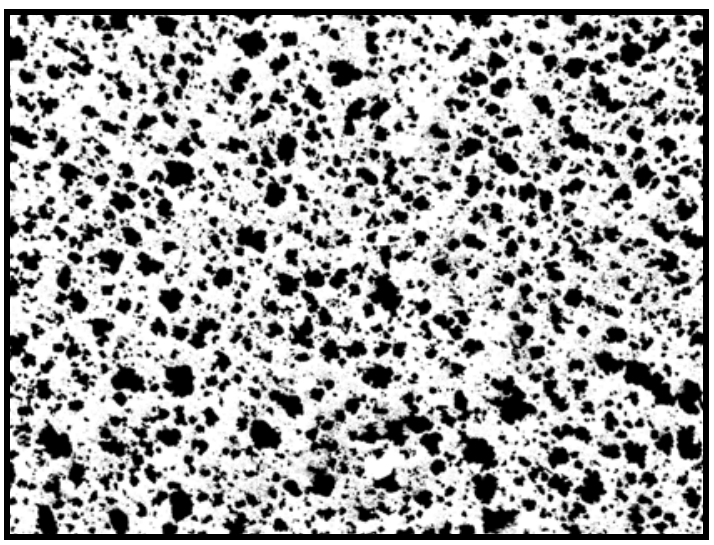

a.

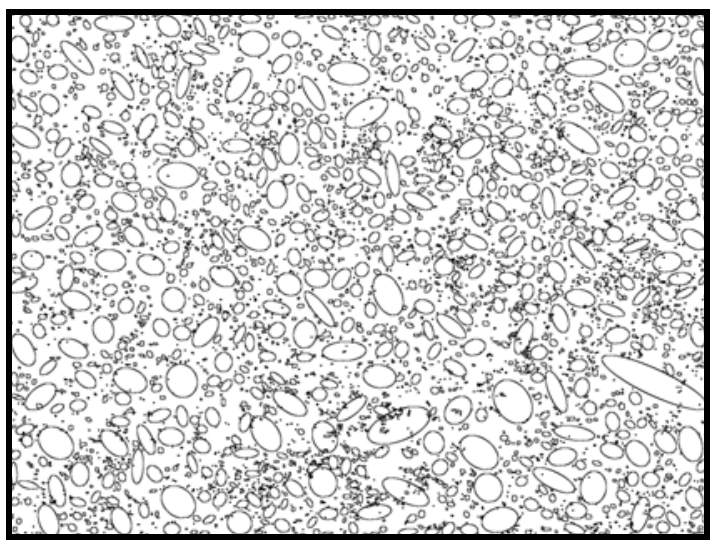

b.

Fig. 6. ATH-PMMA (33\% vol.): a. binary image, and b. fitting ellipse procedure.

Fig. 7 shows histograms of the aspect ratio of the ATH-PMMA composites for $29 \%$ and $49 \%$ vol. fractions. Similar histograms were obtained for the intermediate fractions [4]. The aspect ratio of the particles is in the range from 1 to 7 . A considerable portion of the particles are spherical or nearly spherical with $95 \%$ of the values being below $a=3$. The mean aspect ratio is found to be in the range of 1.7 to 1.86 , the median (middle of the distribution: $50 \%$ of the values above the latter and $50 \%$ of the values below) between 1.53 to 1.8 , and the mode (most frequent value) in the range from 1.31 to 1.39 . 

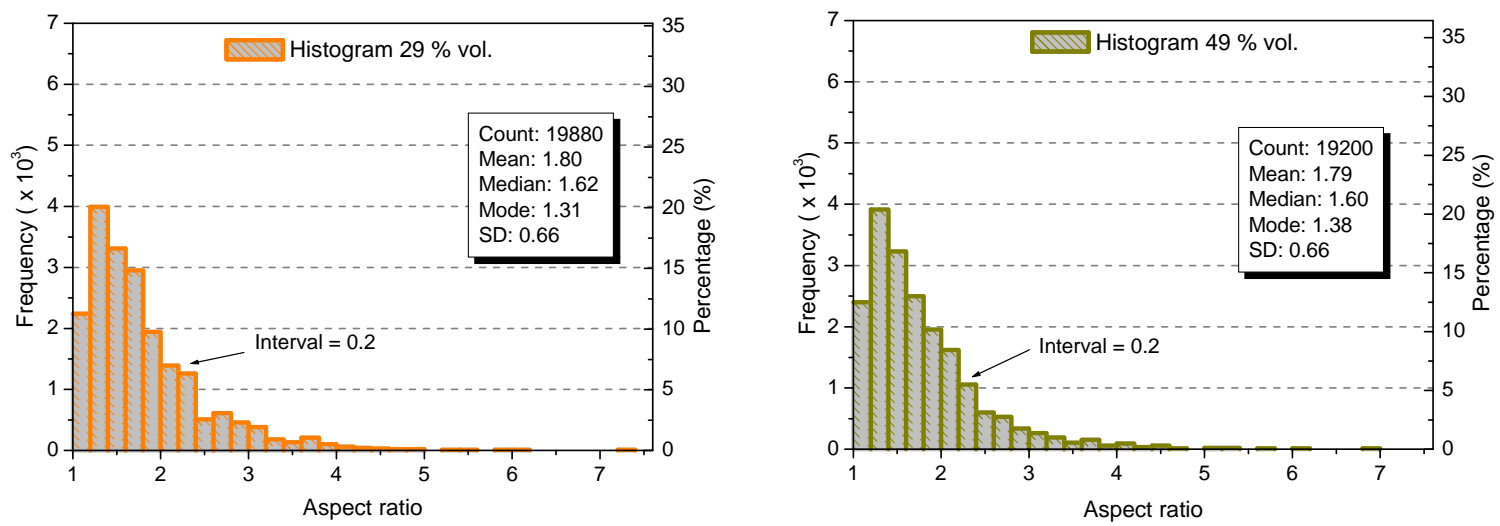

Fig. 7 Histograms of the aspect ratio for ATH-PMMA, $29 \%$ and $49 \%$ vol.

Fig. 8 shows typical SEM micrographs of the ATH powder. The ATH particles resemble aggregates of platelet and prismatic crystals, with sizes varying from around $2 \mu \mathrm{m}$ up to 50 $\mu \mathrm{m}$ or more.

Finally, the laser light scattering results confirmed the average aspect ratio of the ATH particles, giving an average aspect ratio of 1.2 with standard deviation of 6 .
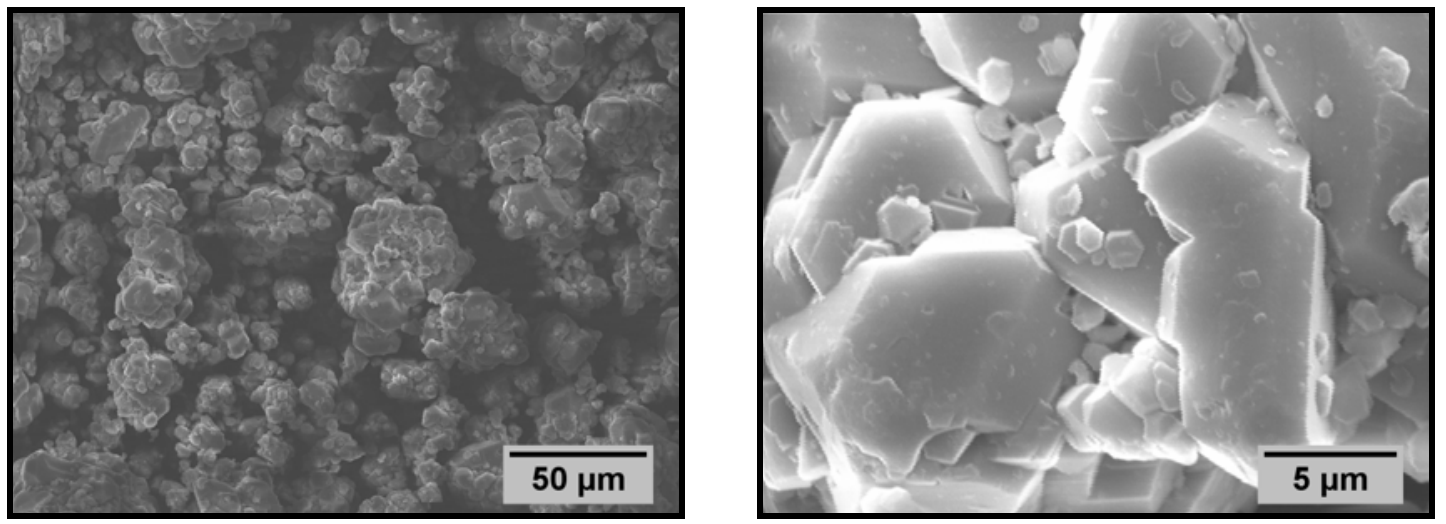

Fig. 8 SEM micrographs of the ATH powder at different magnifications:

\section{Analytical Modelling}

The elastic modulus $E_{m}$ and Poisson's ratio $v_{m}$ of PMMA obtained from the tensile tests at different temperatures (Figs 3 and 4) are used as input in the analytical equations given in section 2. The elastic modulus $E_{f}$ and Poisson's ratio $v_{f}$ of the ATH particles are 
derived from the literature $[29,30]$ with values of $76 \mathrm{GPa}$ and 0.24 respectively. The properties of the filler particles are assumed to be constant with temperature.

Valuable comparison between the theories was achieved by assuming, in the first instance, that the filler particles are spherical, ie $a=1$. The Eshelby tensor $\boldsymbol{S}$ for spherical particles is utilised for the M-T and Lielens models. The shape factor in the $\mathrm{H}-\mathrm{T}$ equations is taken as the one for spherical particles, i.e., $s=2 a=2$. The Nielsen [20] solutions are obtained for the maximum packing of $f_{f \max }=0.74$, suggested for dispersed spheres [21], with no interfacial slip. Fig. 9 shows the predictions of the elastic modulus $E_{c}$ as a function of filler volume fraction $f_{f}$, assuming $a=1$, together with the experimental data for the composites (33\% - 49\% vol.), at room temperature, $21{ }^{\circ} \mathrm{C}$.

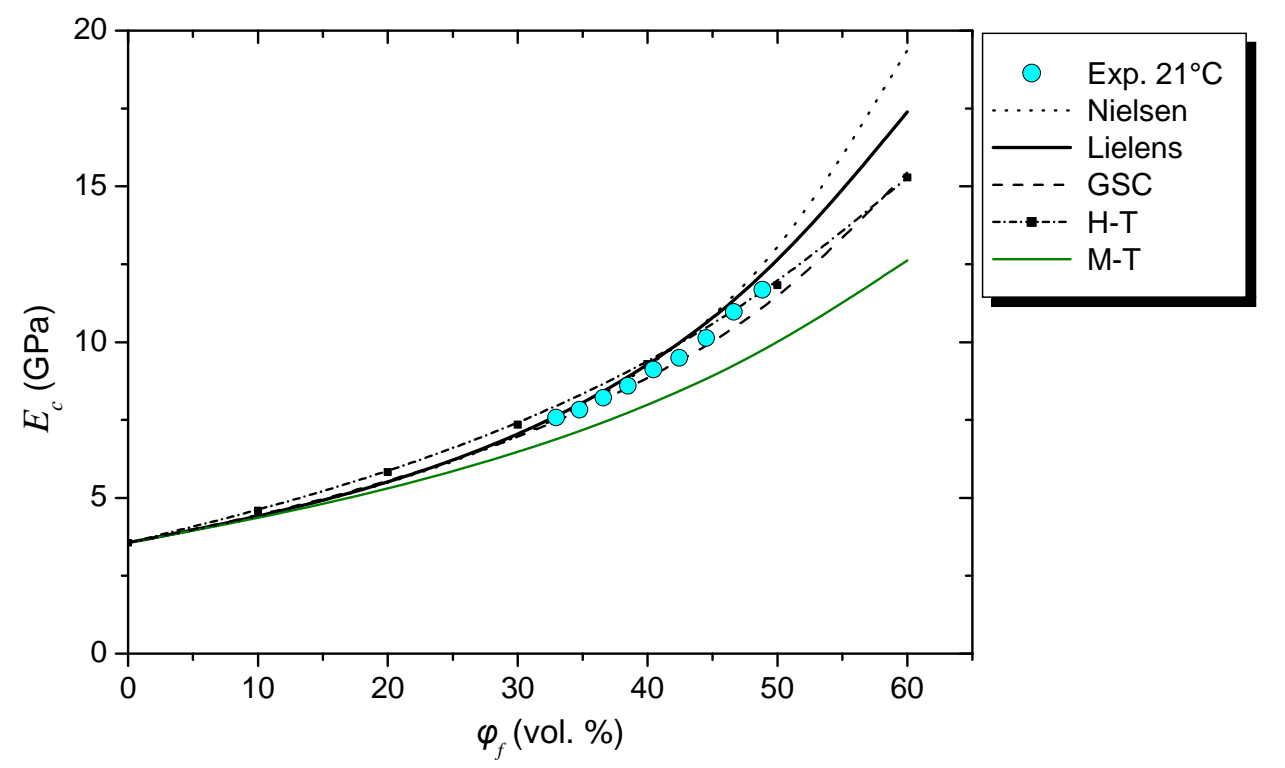

Fig. 9 Comparison of analytical predictions to the experimental data at $21^{\circ} \mathrm{C}$

Very good results are provided by the Nielsen $\left(y_{1}\right)$ and H-T models. These models are the most widely used models for the prediction of the modulus of particle reinforced composites due to their simple form. The GSC model is also found to be in very good agreement with the experimental data. The self consistent equations are not shown since they over-predict the modulus significantly. The M-T model predictions are found to be lower than 
the experimental data as it was derived for relatively low particle concentrations. The Lielens model, which is proposed as an improvement to the M-T model for higher filler volume fractions, provides a superior prediction to the M-T model.

The effect of altering the particle aspect ratio within the range from $1-7$, on the modulus predictions, is next examined for the Lielens and H-T models. The Lielens model allows for two different particle shapes to be modelled: fibre-like and disk-like inclusions. The solutions $E_{1}, E_{2}$, and $E_{3}$, along directions $x_{i}=1,2,3$, for both these shapes are shown in Fig 10, together with the solutions for randomly oriented particles $E_{3 D}$ given by Van Es for fibre-like $E_{3 D \text { fibre }}$ (Eqn. 17), and disk shaped $E_{3 D \text { disk }}$ (Eqn. 18) inclusions. Fig. 10 shows that the aspect ratio has an important effect on the modulus $E_{1}$ for composites with aligned fibrelike inclusions and on the moduli $E_{2}$ and $E_{3}$ for composites with aligned disk-like inclusions, while the effect on the transverse direction is minimal. The random solutions $E_{3 D \text { fiber }}$, and $E_{3 D \text { disk }}$, are intermediately affected by the change in the aspect ratio values with the reinforcing effect being greater for disk-shaped inclusions. The Lielens model ( $E_{3 D}$ fiber or $E_{3 D \text { disk }}$ ) provides an excellent description of the experimental data for the range of aspect ratios from 1 to 2 and good prediction for aspect ratios until about 3; this is in agreement with the values established by the optical microscopy aspect ratio distributions.

The H-T longitudinal, transverse and random Van Es predictions for $s=2 a$ are shown in Fig 11. The predictions (parallel and transverse) for either fibres or disks for $s=2 a$ (Fig 11.a.) are identical and in this case only the random solutions, $E_{3 D \text { fiber }}$ or $E_{3 D \text { disk }}$, differ. For aspect ratios above 2, the H-T model starts to deviate slightly from the experimental data, similarly to the Lielens pattern. 

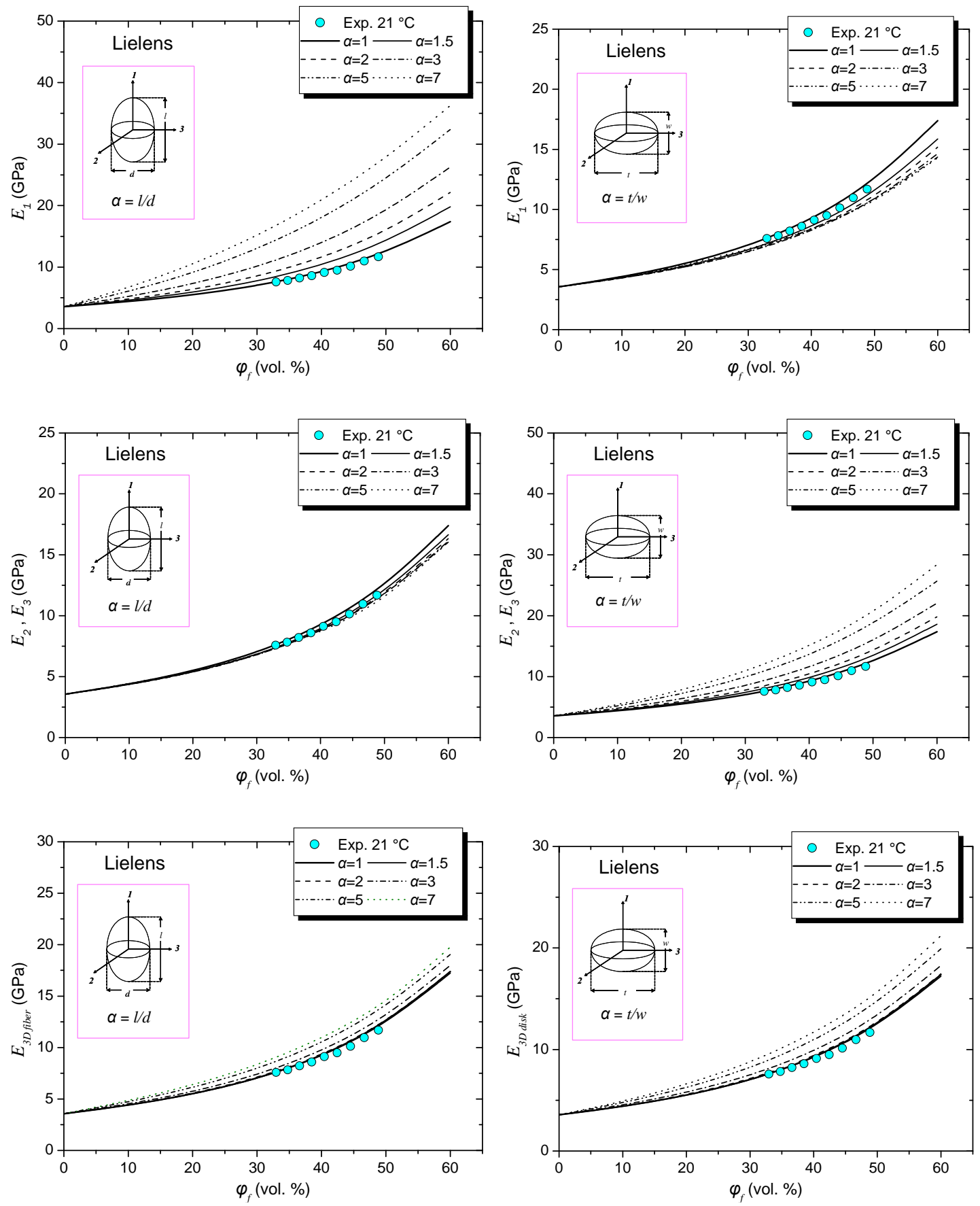

Fig. 10 Lielens model: Effect of aspect ratio on the predictions for fibre-like and disk-like inclusions and comparison with experimental data, at $21^{\circ} \mathrm{C}$. 

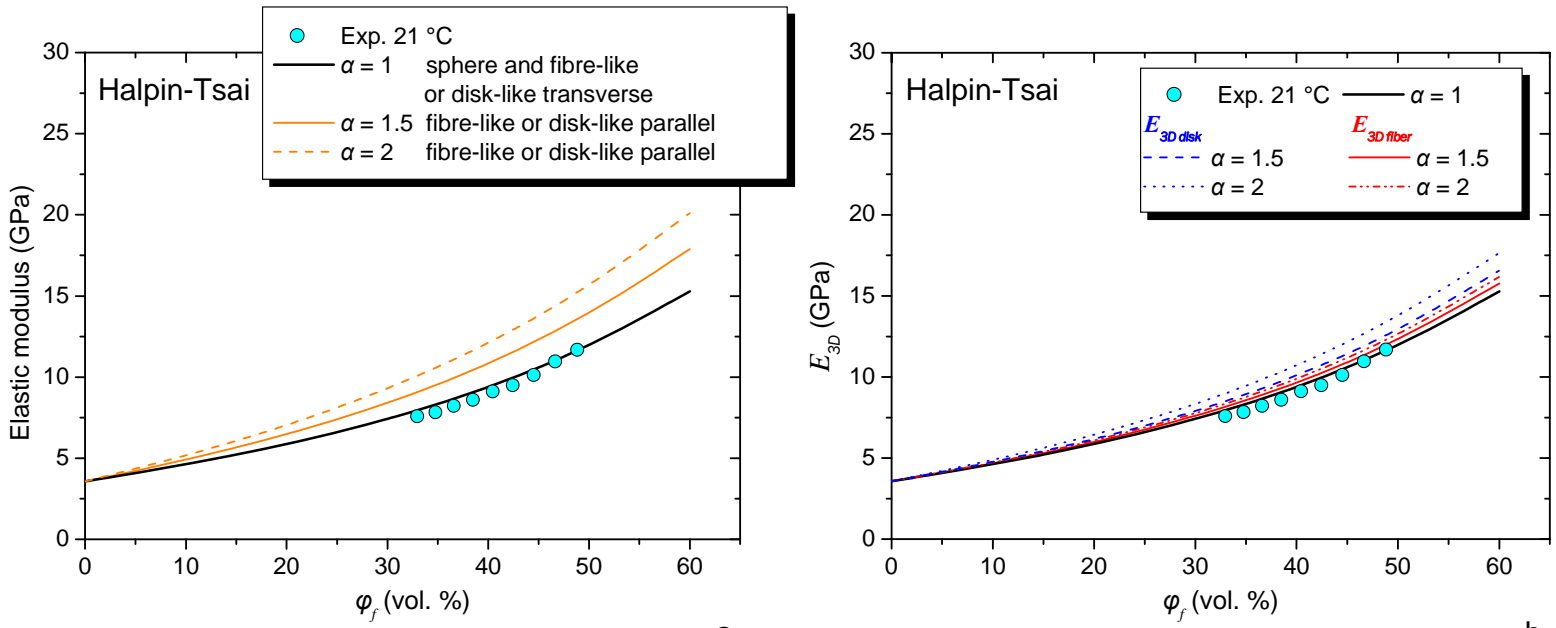

b.

Fig. $11 \mathrm{H}-\mathrm{T}$ model: Effect of $\alpha$ on the predictions for fibre-like and disk-like inclusions.

The Lielens, GSC, H-T and Nielsen $y_{1}$ models are shown against the experimental data at different temperatures in Figs 12 and 13. For simplicity reasons, the aspect ratio of $a=1$ is used. Similar results can be obtained for the random solutions using the Lielens and H-T models for aspect ratios up to 2 . The four models are in very good agreement with the experimental data at all temperatures, with the Lielens and Nielsen models giving an excellent description. The H-T and GSC models slightly deviate from the test data for volume fractions higher than $40 \%$ vol. As mentioned in Section 2 the modification of the H-T model by Lewis \& Nielsen was proposed in order to accommodate higher particle volume fractions into the solutions, so the better agreement in this case was expected.
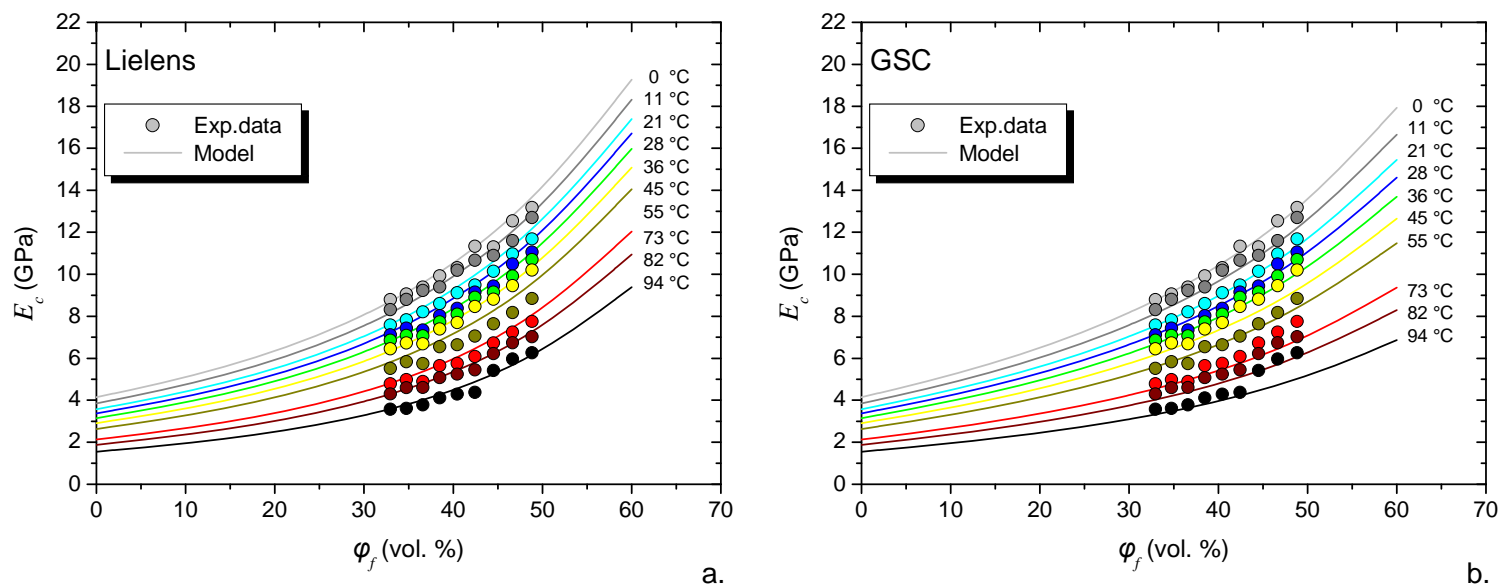
Fig. 12 Elastic modulus against filler volume fraction and temperature: a. Lielens and b. GSC analytical modelling predictions for $a=1$.
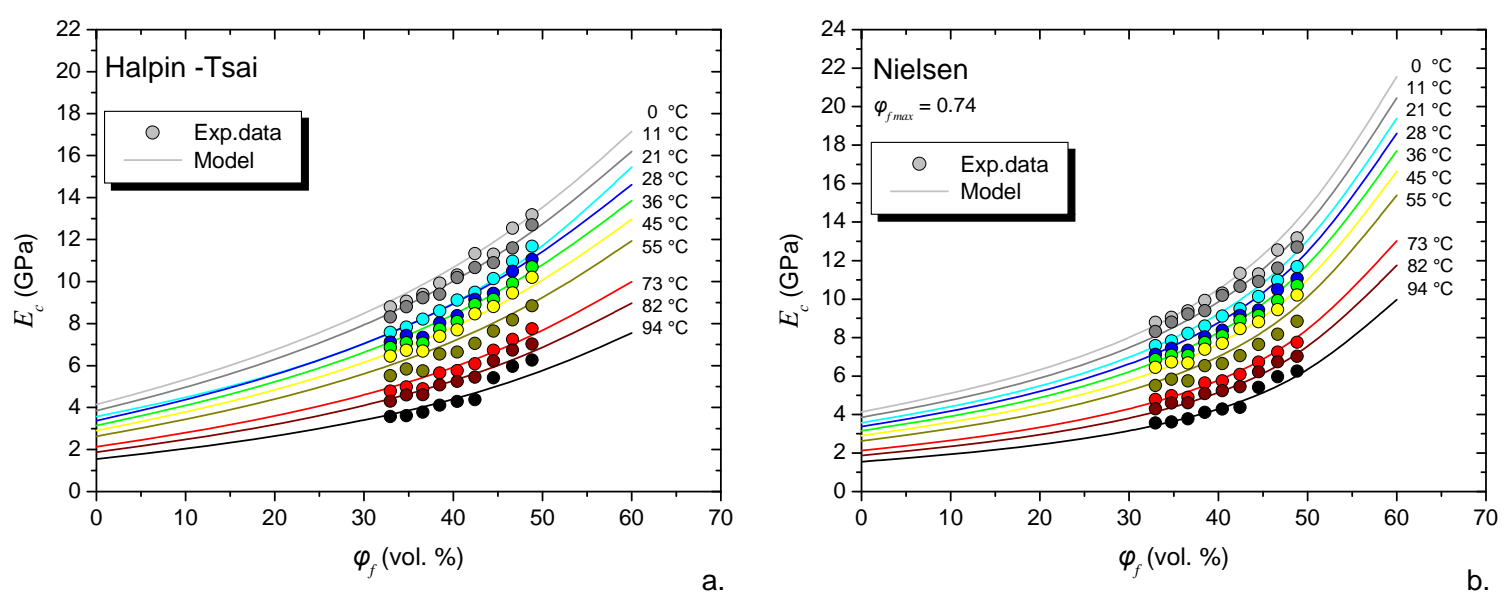

Fig. 13 Elastic modulus against filler volume fraction and temperature: a. Halpin-Tsai, and b. L\&N analytical modelling predictions for $a=1$.

\section{Conclusions}

The modulus of the ATH-PMMA composites increases by $35 \%$ as the ATH filler contents increase from $33 \%$ vol. to $49 \%$ vol., and decreases by $60 \%$ with rising temperature from 0 to $94{ }^{\circ} \mathrm{C}$. This elastic behaviour of the composites is modelled by measuring only the elastic properties of the PMMA matrix and determining the shape of the ATH fillers in the composites via microscopy. Under the assumption of constant filler elastic properties and by altering the temperature from 0 to $94{ }^{\circ} \mathrm{C}$, it is as if at every temperature a different composite is tested as the matrix material softens.

The Lielens model is the most representative of the elastic behaviour of the ATHPMMA composites. The predicted values are in excellent agreement with the experimental data at different temperatures and various filler contents. It provides an exceptional description of the experimental data for the range of aspect ratios from 1 to 2 and good prediction for aspect ratios until about 3; values that represent the ATH particles precisely. 
Moreover, the Lielens model is based on constitutive modelling equations and can be used to predict all the elastic constants of the composites at the same time. This makes it superior to the other modelling equations, i.e., the Halpin-Tsai, GSC and Nielsen models which involve a high degree of empiricism.

\section{Acknowledgements}

The authors would like to acknowledge E. I. DuPont Nemours \&Co. (Inc.) for funding this work and supplying the materials.

\section{References}

1.) Duggins, R. B., U.S. Patent 3,847,865, 260/42.52, 1974

2.) Tucker III, C. L. and Liang, E., Compos Sci Technol, vol. 59, 655-671, 1999

3.) Fu, S-Y., Feng, X-Q., Lauke, B. and Mai, Y-W., Composites Part B, 39, 933-961, 2008.

4.) Stapountzi O. A., PhD thesis, Stifffness and fracture properties of Alumina Trihydrate filled Poly (Methyl Methacrylate) composites, Imperial College London, April 2008

5.) Hill, R., J Mech Phys Solids, vol. 11, 357-372, 1963

6.) Eshelby, J. D., Proc Roy Soc A, vol. 241, 376-396, 1957

7.) Mura, T., Micromechanics of Defects in Solids, The Hague: Martinus Nijhoff, 1982

8.) Hashin, Z. and Shtrikman, S., J Mech Phys Solids, vol. 11, 127-140, 1963

9.) Mori, T. and Tanaka, K., Acta Metallurgica, vol. 21, 571-574, 1973

10) McCullough, R. L., In: Micromechanical materials modelling, Delaware Composites

Design Encyclopedia - Volume 2, Whitney, J. M. and McCullough, R. L., editors, Technomic Pub. Co, Lancaster, PA, 1990

11.) Hill, R., Proc Phys Soc A, vol. 65, 349-354, 1952

12.) Hill R., J Mech Phys Solids, vol. 12, 199-212, 1964

13.) Weng, G. J., Int J Eng Sci, vol. 30, 83-92, 1992 
14.) Lielens, G., Pirotte, P., Couniot, A., Dupret, F., Keunings, R., Composites A, vol. 29, 670,1997

15.) Hill, R., J Mech Phys Solids, vol. 13, 213-222, 1965

16.) Kerner, E. H., Proc Phys Soc B, vol. 69, 808-813, 1956

17.) Hermans, J. J., Proc Kon Ned Akad v Wetensch B, vol. 65, 1-9, 1967

18.) Halpin, J. C., J Compos Mater, vol. 3, 732-734, 1969

19.) Halpin, J. C. and Kardos, J. L., Polymer Eng Science, vol. 16, 344-352, 1976

20.) Lewis, T. B, Nielsen, L. E., J Appl Poly Sci, vol. 14, 1449-1471, 1970

21.) Nielsen, L. E. and Landel, R. F., Mechanical properties of polymers and composites, CIP, 1994

22.) Van Es, M., Xiqiao, F., Van Turnhout J., Van der Giessen, E., Al-Malaika S, Golovoy AW, editors, Specialty polymer additives: principles and applications, CA Malden, MA Blackwell Science, 2001

23.) ASTM D790M-93, Annual Book of ASTM Standard, American Society for Testing and Materials, 1993

24.) ASTM D638M-84 Standard test method for tensile properties of plastics, 1993

25.) ImageJ for Microscopy, MBF ImageJ/ v1.38f -1.39d, User Manual, 2007

26.) Malvern Instruments 2000 (http://www.malvern.co.uk)

27.) Ferreira, J. M., Costa, J. D., Capela, C., Theor Appl Fract Mech, vol. 26, 105-116, 1997

28.) Zoller, P. and Walsh, D., Standard pressure-volume-temperature data for polymers,

Technomic Pub. Co., 1995

29.) Nie, S. and Basaran, C., Int J Solid Struct, vol. 42, 4179-4191, 2005

30.) Liu, H., Hu, J., Xu J., Liu, Z., Shu, J., Mao, H. K. and Chen, J., Phys Chem Miner, vol.

$31,240-246,2004$ 